\title{
Correction to: Risk Factors for Clostridium difficile Isolation in Inflammatory Bowel Disease: A Prospective Study
}

\author{
Dejan Micic ${ }^{1}$ - Andres Yarur ${ }^{1}$ - Alex Gonsalves ${ }^{1} \cdot$ Vijaya L. Rao $^{1}$ - Susan Broadaway ${ }^{2} \cdot$ Russell Cohen $^{1}$ - Sushila Dalal ${ }^{1}$. \\ John N. Gaetano ${ }^{1}$ - Laura R. Glick ${ }^{1}$ - Ayal Hirsch ${ }^{1}$ - Joel Pekow ${ }^{1}$ - Atsushi Sakuraba ${ }^{1}$ - Seth T. Walk ${ }^{2}$. David T. Rubin ${ }^{1}$
}

Published online: 22 August 2018

๑) Springer Science+Business Media, LLC, part of Springer Nature 2018

\section{Correction to: \\ Digestive Diseases and Sciences (2018) 63:1016-1024 \\ https://doi.org/10.1007/s10620-018-4941-7}

The original version of the article unfortunately contained an error in a percentage value in Results section of Abstract.

The sentence "Mixed infection with more than one toxigenic isolate was present in 5/16 (33.3\%) individuals." should be "Mixed infection with more than one toxigenic isolate was present in 5/16 (31.3\%) individuals."

The original article can be found online at https://doi.org/10.1007/ s10620-018-4941-7.

David T. Rubin

drubin@medicine.bsd.uchicago.edu

Dejan Micic

Dejan.micic@northwestern.edu

Andres Yarur

ajyarur@gmail.com

Alex Gonsalves

alex@gonsalves.com

Vijaya L. Rao

Vijaya.1.rao@gmail.com

Susan Broadaway

scb@montana.edu

Russell Cohen

rcohen@medicine.bsd.uchicago.edu

Sushila Dalal

Sushila.Dalal@uchospitals.edu

John N. Gaetano

jngaetano@northwestern.edu
Laura R. Glick

lglick@uchicago.edu

Ayal Hirsch

ayal.red@gmail.com

Joel Pekow

jpekow@bsd.uchicago.edu

Atsushi Sakuraba

asakurab@medicine.bsd.uchicago.edu

Seth T. Walk

seth.walk@montana.edu

1 University of Chicago Medicine Inflammatory Bowel Disease Center, 5841 South Maryland Avenue, MC4076, Chicago, IL 60637, USA

2 Montana State University, Bozeman, MT 59717, USA 